
\title{
The Partial Beam Lifetime at RHIC due to Coulomb
}

\author{
M. J. Rhoades-Brown and J. Weneser
}

July 1992

\section{DISCLAIMER}

This report was prepared as an account of work sponsored by an agency of the United States Weither the United States Government nor any agency thereof, nor any of their Government. Neither the United States employees, makes any warranty, express or implied, or assumes any legal habitity or the accuracy, completeness, or usefulness of any information, apparatus, produch, or process disclosed, or represents that its use would not infringe psivately' owned rights. Reference herein to any specific cornmercial product, process, or service by tiade norse, lradent, recommanufacturer, or ctherwise does not necessarily constifute or imply its endotsement, The views mendation, or favering by the United States Government or any agency lhereof The views and opinions of authors expressed herein do not necessarily state or teflect those of the United States Govtrnment or any agency thereof.

\section{RHIC P R O E C T}

\author{
Brookhaven National Laboratory \\ Associated Universities, Inc. \\ Upton, NY 11973
}


The partial beam lifetime at RHIC duc to Coulomb dissociation of the nucleus

\author{
M.J. Rhoades-Brown and J. Weneser" \\ RHIC Project and Physics Department* \\ Brookhaven National Laboratory
}

\title{
Introduction
}

During beam crossing at RHIC, the Lorentz contracted Coulomb interaction between the heavy ions will excite internal modes of the nucleus. The subsequent decay of these modes is predominately via single or multiple nucleon emission. Changing the atomic mass of the beam ion will eventually cause beam intensity loss at RHIC for the radius of the ion orbit is sensitive to changes of the ratio $\mathrm{Z} / \mathrm{A}$.

While calculations for this beam loss mechanism have been made ${ }^{1}$, it is now clear that these earlier theoretical studies underestimated the Coulomb dissociation loss rate for they appear to have included only a limited range (first dipole resonance region, up to $\sim 30$ $\mathrm{MeV}$ ) of internal nuclear excitation energy.

In this report we reexamine the question of Coulomb dissociation cross sections at RHIC by including internal excitation energies up to thousands of GeV. In addition, we utilize experimental photonuclear absorption cross sections when evaluating the dissociation cross section. Also, internal excitation of a nucleus in one beam will result in both energy loss and transverse momentum change of an ion in the colliding bearn. These recoil effects will be examined in detail to determine if there is an additional loss rate for ions out of the rf bucket or a non-negligible change in the ion's betatron momentum.

\section{Coulomb Dissociation Cross Section}

The Coulomb dissociation cross section is given by the Weizsacker-Williams expression (whose accuracy is explored in Ref. 2)

$$
\sigma=\frac{2 a Z_{p}^{2}}{\pi(\hbar c)^{2} \gamma_{e f f}^{2}} \int d \omega \omega \sigma_{p h}(\omega) \int_{b_{\min }}^{\infty} b d b K_{1}^{2}\left(\left|\frac{\omega \omega}{h c \gamma_{e f f}}\right|\right)
$$

where $Z_{p}$ is the ion atomic number, $\gamma_{e f f}$ is the fixed target frame Lorentz parameter ( $\gamma_{\text {eff }}=2 \gamma^{2}-1, \gamma=108$ for ${ }^{197} A u^{79+}$ at RHIC), $\alpha$ is the fine structure constant, $K_{y} \cdot$ is a Macdonald or modified Bessel function, $b$ is the impact parameter between the two 
colliding ions, and $\sigma_{p h}(\omega)$ is the target ion photonuclear cross section at photon energy $\omega$ $\mathrm{MeV}$. In this report the values of $\sigma_{p h}(\omega)$ are taken from experimental data.

Equation (1) is an expression for the perturbative transition amplitude squared that is correct $^{2}$ to order $1 / \gamma_{\text {eff }}^{2}$. The cut off in impact parameter is determined by the asymptotic behavior of $K_{1}(z)$. For $z \gg 1, K_{1}(z)$ drops faster then exponentially $\left[\right.$ as $\left.(\pi / 2 z)^{1 / 2} \mathrm{e}^{-z}\right]$. The integration over $b$ was carried out analy tically using the expression,

$$
\int^{z} t K_{1}(k t) K_{1}(k t) d t=\left(z^{2} / 2\right)\left[K_{1}^{2}(k z)-K_{0}(k z) K_{2}(k z)\right]
$$

In all results presented here, the lower integration limit $b_{\min }$ was taken to be $15 \mathrm{fm}$, i.e. the grazing impact parameter between two $A u$ ions. The dependence on $b_{\min }$ is weak.

The experimental values for $\sigma_{p h}(\omega)$ were taken from several sources. Starting with the nuclear separation energy of $8 \mathrm{MeV}, \sigma_{p h}(\omega)$ for $\mathrm{Au}$ ions and the range $8 \mathrm{MeV} \leqslant$ $\omega \leq 28 \mathrm{MeV}$ were taken from the measurements of Veyssiere ${ }^{3}$ et al. For the range 28 $\mathrm{MeV} \leq \omega \leq 103 \mathrm{MeV}$, the work of Lepretre et al. was used. This data is for ${ }^{208} \mathrm{~Pb}$, and $\sigma_{p h}$ was scaled as 197/208 to obtain $\mathrm{Au}$ values. For the range $103 \mathrm{MeV} \leq \omega \leq 440$ $\mathrm{MeV}$ the scaled values ( $\mathrm{Pb}$ data) of $\mathrm{Carlos}^{5}$ et al. were used. For $\omega$ values in the range $440 \mathrm{MeV} \leq \omega \leq 2 \mathrm{GeV}$ there are no experimental measurements of $\sigma_{p h}$ for Au. In this region scaled values of the $\gamma, p$ and $\gamma$, $n$ experimental cross section ${ }^{6}$ were utilized, i.e., $\sigma_{p h}(\omega)=Z_{T} \sigma_{\gamma, p}(\omega)+\left(A_{T}-Z_{T}\right) \sigma_{\gamma, n}(\omega)$. For the range $2 \mathrm{GeV} \leq \omega \leq 9.51$ GeV the Au data of Michalowski ${ }^{7}$ et al. was used. For the range $9.51 \mathrm{GeV}<\omega<17.84$ GeV the scaled values of the $\gamma, \mathrm{p}$ and $\gamma, \mathrm{n}$ experimental cross sections ${ }^{6}$ were once again utilized. Beyond the range of the table ${ }^{6}(\omega>17.84 \mathrm{GeV})$ it was assumed that the $\gamma, p$ and $\gamma$, n cross sections are constant in $\omega$, with a value taken from the tables at $17.84 \mathrm{GoV}$. Inspection of the tabulated data between 10 and $17 \mathrm{GeV}$ indicates that this is a very reasonable assumption.

We note that using scaled $\gamma, \mathrm{p}$ and $\gamma, \mathrm{n}$ datit for the range $440 \mathrm{MeV} \leq \omega \leq 2 \mathrm{GeV}$ underestimates the $\sigma_{p h}(\omega)$ cross section for An. Comparing with known Aumeasurements at $440 \mathrm{MeV}$ and $2 \mathrm{GeV}$ suggests an underestimate in the contributions from this $\omega$ region of approximately 15\%. By contrast, studies by Michalowski at al. indicate that for w values greater than $9.51 \mathrm{GeV}$ it is necessary to multiply $\sigma$ by a shadowing factor ${ }^{7} \mathrm{~S}$, i.e. a factor to take into account the fact that not all nucleons contribute to the photomuclear 
cross section. For Au ions with $\omega>9.51 \mathrm{GeV}$ wo have taken a value ${ }^{7}$ for $\mathrm{S}$ of 0.75 . We also note; that except for the regions $8 \mathrm{MeV} \leq \omega \leq 12 \mathrm{MeV}$ and $28 \mathrm{MeV} \leq \omega \leq 103 \mathrm{MeV}$, the photonuclear cross sections were given as tabulated experimental data. When actual tabulated data was unavailable, it was necessary to read published graphs.

In Table I, the relative contributions to $\sigma$ are given for different ranges of $\omega$.

The error estimates for $\sigma(b)$ were calculated from equation (1) using the experimental errors for the photonuclear cross sections. For the $\omega$ range $\omega>9.51 \mathrm{GeV}$, the overall uncertainties in the extrapolation suggested an error value of $1.0 \mathrm{~b}$.

Table 1: Relative Contributions to $\sigma$

\begin{tabular}{|rll|c|}
\hline \multicolumn{3}{|c|}{$\omega$ Range $(\mathrm{MeV})$} & $\sigma(\mathrm{b})$ \\
\hline 8 & $\leq \omega \leq$ & $\leq 1.5 \pm 6.1$ \\
28 & $\leq \omega \leq 103$ & $4.9 \pm 0.8$ \\
103 & $\leq \omega \leq 440$ & $12.4 \pm 0.6$ \\
440 & $\leq \omega \leq 2000$ & $8.5 \pm 1.0$ \\
2000 & $\leq \omega \leq 9510$ & $2.7 \pm 0.08$ \\
9510 & $\leq \omega \leq 25000$ & $2.3 \pm 1.0$ \\
\hline
\end{tabular}

It can be guessed from this table where the quoted value ${ }^{1}$ of $60 \mathrm{~b}$ came from. Including higher excitation energies gives a total value of $\sigma=(92.3 \pm 6.3) \mathrm{b}, \mathrm{a} \sim 54 \%$ increase. The total error was calculated from a sum of squares analysis of the errors in Table 1.

Momentum Recoil out of the if bucket

Including higher internal excitation energies in one nucleus, it is necessary to consider possible beam loss due to momentum recoil of the other colliding ion out of the of bucket.

We label the two ions as 1 and 2 , and use $X$ to denote the laboratory or colliding frame. If $\Delta \mathrm{E}$ represents the energy loss of ion 2 in frame $\mathrm{X}$,

$$
\begin{aligned}
& E_{2} \rightarrow E_{2}+\Delta E \\
& p_{2} \rightarrow p_{2}+\frac{\partial p}{\partial E} \Delta E
\end{aligned}
$$

Hence in the frame of ion 1 (denoted prime),

$$
\Delta E_{2}^{\prime}+E_{2}^{\prime}=\gamma\left[\left(E_{2}+\Delta E-\beta\left(-p_{2}-\frac{\partial p}{\partial E} \Delta E\right)\right)\right]
$$

or 


$$
\begin{gathered}
\Delta E_{2}^{\prime}=\gamma\left[\Delta E+\frac{\partial p}{\partial E} \beta \Delta E\right] \\
=2 \gamma \Delta E
\end{gathered}
$$

where the relation $\beta=p / E$ (momentum of ion $1, p_{1}^{\prime} \equiv 0$ ) has been used.

Hence, if we assume an if voltage of $6.7 \mathrm{MV}$, this gives a bucket area ${ }^{8}$ of $1.31 \mathrm{eV} \mathrm{sec} / \mathrm{u}$ and a $\Delta E / E$ value for the bucket of $\Delta E / E=2 \times 10^{-3}$. Hence for $100 \mathrm{GeV} / \mathrm{u}^{197} \mathrm{Au}{ }^{79}$ beams at RHIC, equation (6) gives

$$
\Delta E_{2}^{\prime}=851 G e V
$$

as the value of energy transfer beyond which the recoiling ion will be lost.

It is obvious from both the tabulated photonuclear cross sections ${ }^{6}$, and the cut-off properties of $K_{1}(z)$ discussed earlier, that partial cross section contributions for $\omega \geq 851$ $\mathrm{GeV}$ will be negligible. Recoil of an ion out of the of bucket following Coulomb excitation of the colliding ion will not occur appreciably. Momentum Recoil Effects on Betatron Motion

During beam crossing at RHIC, the r.m.s. value for the transverse component of the betatron momentum $\left\langle p_{\perp}\right\rangle_{r m s}$ is related to the longitudinal component of momenta $p_{\|}$ via,

$$
<p_{\perp}>_{r m s}=\frac{1}{\sqrt{2}} \sqrt{\frac{E_{N}}{\pi(\beta \gamma) \beta^{*}}} p_{\|}
$$

where $E_{N}$ is the normalized transverse emittance (taken to be $10 \pi \times 10^{-6} \mathrm{~m}$ rad) and $\beta^{*}$ is the value of the beta function at the beam crossing point (taken to be $2 \mathrm{~m}$ ). With these values,

$$
\left\langle p_{\perp}\right\rangle_{r m s}=1.5 \times 10^{-4} p_{\|} \equiv 3000 \mathrm{MeV}
$$

where for $\mathrm{Au}$ ions $\mathrm{p}_{\|}=100 \mathrm{GeV} \mathrm{A} / \mathrm{c}$.

For Coulomb excitation of the ions, the energy transfer between the ions corresponds almost entirely to a longitudinal momentum transfer. The transverse momentum (as given by perturbation theory in the appendix) is of the order $\omega / \gamma_{e f f}$. Since $\omega \leq 25$ GeV and 
$\gamma \sim 2.3 \times 10^{4}$, the accompanying transverse momentum push is of the order $10^{-3} \mathrm{GeV}$ or less. This value is well within the betatron momentum aperture and hence we can assume the recoil accompanying Coulomb excitation to have a negligible effect on the betatron motion of the ions.

\section{Partial Lifetime at RHIC}

Using the values of luminosity in the Design Manual, ${ }^{1,9}$ i.e., $\mathrm{L}=8.40 \times 10^{26} \mathrm{~cm}^{-2}$ $\mathrm{sec}^{-1}$, the intensity decay rate $\lambda_{3}$ for Coulomb dissociation of $\mathrm{Au}$ beams is now modified from $19 \times 10^{-3} \mathrm{~h}^{-1}$ to a value

$$
\lambda_{3}=(29.2 \pm 2.0) \times 10^{-3} h^{-1}
$$




\section{References}

1. Conceptual Design of the Relativistic Heavy Ion Collider RHIC, p. 119, May 1989, BNL 52195.

2. A.J. Baltz, M.J. Rhoades-Brown, J. Weneser, Phys. Rev. A444 5569 (1991).

3. A. Veyssiere, H. Beil, R. Bergere, P. Carlos and L. Lepretre, Nucl. Phys. A159, 561 (1970).

4. A. Lepretre, H. Beil, R. Gergere, P. Carlos, J. Fagot, A. De Miniac and A. Veyssiere, Nucl. Phys. A367, 237 (1981).

5. P. Carlos, H. Beil, R. Bergere, J. Fagot, A. Lepretre, A. De Miniac and A. Veyssiere, Nucl Phys. A431, 573 (1984).

6. Total cross-sections for reactions of high energy particles, Volume 12, Landolt- Bornsein, edited by H. Schopper.

7. S. Michalowski, D. Andrews, J. Eickmeyer, T. Gentile, N. Mistry, R. Talman, K. Ueno, Phys. Rev. Lett. 199,737 (1977).

8. H. Hahn, private communication.

9. In Ref. (1), p. 117-119, it is stated that a luminosity value for Au ions from Table IV. 3-1 was used in estimating the beam loss rate. Two values are given in Table IV. $3-1, \mathrm{~L}=2 \times 10^{26} \mathrm{~cm}^{-2} \mathrm{sec}^{-1}$ and $\mathrm{L}=1.1 \times 10^{27} \mathrm{~cm}^{-2} \mathrm{sec}^{-1}$. However, closer inspection of the loss rates quoted indicates a luminosity value of $\mathrm{L}=8.40 \times 10^{26} \mathrm{~cm}^{-2} \mathrm{sec}^{-1}$ was used. Of course, a self-consistent analysis of time dependent luminosity and beam loss rate that includes in ra-beam scattering is required. 


\section{APPENDIX}

In order to calculate the transverse recoil momentum given to the projectile ion it is useful to re-work the Weizsecker-Williams cross section so as to make explicit the momentum transfers. Distinguishing between projectile ion and target ion by the subscripts $P, T$, we can write the transverse-photon transfer cross section:

$$
\frac{d \sigma}{d \Omega}=2 \pi \int d E_{F} \frac{p_{P_{j}} E_{P_{f}}}{(2 \pi \hbar)^{3}} \frac{1}{v_{p_{0}}}\left|\left\langle p_{P_{j}}\left|\vec{j} \cdot \hat{e} e^{-i q \cdot r_{P}}\right| p_{P_{0}}\right\rangle\right|^{2} \frac{(4 \pi)^{2}}{\left[\vec{q}^{2}-q_{0}^{2}\right]^{2}}\left|\left\langle T_{f}\left|\vec{j} \cdot \hat{e} e^{i \vec{q} \cdot \vec{r}_{T}}\right| T_{0}\right\rangle\right|^{2}
$$

The $\vec{q}$ represents the momentum transfer, $\vec{q}=\vec{p}_{P_{0}}-\vec{p}_{P_{f}} ; q_{0}$ is the corresponding energy transfer, $\left(E_{P_{0}}-E_{P_{f}}\right)=\omega$. It is assumed here that the projectile ion is not internally excited, and that it is sufficient to lepresent the initial and final projectile states as plane waves. The target states need not be explicitly represented since the target matrix element will be swept into the effective photon cross section as in the usual Weizsacker-Williams approximations. It can be shown (not here) that the Coulomb or longitudinal photon contribution is of lower order in $M_{P} / E_{P}=1 / \gamma$, and that the transverse part outlined above suffices for the leading $(\ln \gamma)$ approximation.

The matrix element for the projectile transition, taken as that of a spinless particle, leads to the vertex strength

$$
\begin{aligned}
\sum_{\hat{c}}\left|\left\langle p_{P_{f}}\left|\vec{j} \cdot \hat{e} e^{-i q \cdot r_{p}}\right| p_{P_{0}}\right\rangle\right|^{2} & =\alpha Z_{p}^{2} \sum\left|\frac{\overrightarrow{p_{P_{f}}+p_{P_{0}}} \cdot \hat{e}}{2 \sqrt{E_{P_{f}} \cdot E_{P_{0}}}}\right|^{2} \\
& =\frac{\left(\overrightarrow{p_{P_{f}}+p_{P_{0}}}\right)^{2}-\left(\left(\overrightarrow{p_{P_{f}}+p_{P_{0}}}\right) \cdot \vec{q}\right)^{2} / q^{2}}{4 E_{P_{0}} E_{P_{f}}} \\
& =\frac{2\left(p_{P_{0}}+p_{P_{f}}\right)^{2}}{E_{P_{0}} E_{P_{f}}(\vec{q})^{2}}\left(\chi-\chi^{2} / 2\right), \\
\chi & =1-\cos \theta=\theta^{2} / 2+\ldots ;
\end{aligned}
$$

$\theta$ is the projectile scattering angle. For high energies and relatively small energy losses this is

$$
\cong \frac{2 p_{p_{0}}}{q^{2}}\left(\chi-\chi^{2} / 2\right)
$$


Similarly

$$
\begin{aligned}
\vec{q}^{2} & =\left(\overline{p_{P_{0}}-}-\overrightarrow{p_{P_{f}}}\right)^{2}=\left(p_{P_{0}}-p_{P_{f}}\right)^{2}+2 p_{P_{0}} p_{P_{f}} \chi \\
& =2 p_{P_{0}} p_{P_{0}} \chi+\omega^{2}+\omega^{2} \frac{M_{P}^{2}}{p_{P_{0}}^{2}}+\left(\text { higher order in } 1 / p_{P_{0}}\right) \\
\vec{q}^{2}-q_{0}^{2} & =2 p_{P_{0}} p_{P_{f}} \chi+\omega^{2} \frac{M_{P}^{2}}{p_{P_{0}}^{2}}+\left(\text { higher order in } 1 / p_{P_{0}}\right) \\
& \cong 2 p_{P_{0}} p_{P_{f}} \chi+\omega^{2} / \gamma^{2} \\
\vec{q}^{2} & \cong 2 p_{P_{0}} p_{P_{f}} \chi+\omega^{2}, \quad \gamma \gg 1 .
\end{aligned}
$$

This allows us to write $d \sigma / d \Omega$ as:

$$
\frac{d \sigma}{d \Omega}=8 \alpha Z_{p}^{2} \int d E_{P_{f}} \frac{p_{P_{f}} E_{P_{f}}}{v_{p_{0}}} \frac{p_{P_{0}}^{2}\left(\chi-\chi^{2} / 2\right)}{\left[\omega^{2}+2 p_{P_{0}} p_{P_{f}} \chi\right]\left[\omega^{2} / \gamma^{2}+2 p_{P_{0}} p_{P_{f}} \chi\right]^{2}}\left|\left\langle T_{f}\left|j_{\perp} e^{i \vec{q} \cdot \vec{r}_{T}}\right| T_{0}\right\rangle\right|^{2}
$$

The equivalent of the Weizsacker-Williams approximation is made by noting that the denominator factor, $\left[\omega^{2} / \gamma^{2}+2 p_{P_{0}} p_{P_{0}} \chi\right]^{2}$, weights $\chi$ so that $\left(2 p_{P_{0}} p_{P_{f}} \chi\right)$ is of order $\omega^{2} / \gamma^{2}$. Then, $\vec{q}^{2}$ differs from $\omega^{2}$ only by the negligible quantity $\omega^{2} / \gamma^{2}$. This, in turn, means that, to this order, the intermediate virtual photon is on the mass shell; that is, since $|\vec{q}| \simeq \omega$, the matrix element $\left\langle T_{f}\left|j_{\perp} e^{i \vec{q} \cdot \vec{r}_{T}}\right| T_{0}\right\rangle$ is well approximated by the real photon matrix element and we can take over from experiment the photo cross sertion:

$$
\sigma_{\gamma}\left(T_{o} \rightarrow T_{F}\right)=\frac{4 \pi^{2}}{\omega}\left|\left\langle T_{f}\left|j_{\perp} e^{i \vec{q} \cdot \vec{r}_{T}}\right| T_{0}\right\rangle\right|^{2}
$$

Then

$$
\frac{d \sigma}{d \Omega}=8 \alpha Z_{p}^{2} \int d E_{P_{f}} \frac{p_{P_{f}} E_{P_{f}}}{v_{p_{0}}} \frac{p_{P_{0}}^{2}\left(\chi-\chi^{2} / 2\right)}{\left[\omega^{2}+2 p_{P_{0}} p_{P_{f}} \chi\right]\left[\omega^{2} / \gamma^{2}+2 p_{P_{0}} p_{P_{f}} \chi^{\chi}\right]^{2}} \frac{\omega}{4 \pi^{2}} \sigma_{\gamma}\left(T_{0} \rightarrow T_{F}\right) .
$$

Then, with the necessary neglect of $\chi^{2} / 2, p_{P_{f}} \simeq E_{P_{\mathcal{f}}} \simeq p_{P_{0}}, v_{p_{0}} \sim 1$ :

$$
\begin{aligned}
\sigma & =\frac{4}{\pi} p_{P_{0}}^{4} \alpha Z_{p}^{2} \int d E_{P_{f}} \omega \int d \chi \frac{\chi}{\left[\omega^{2}+2 p_{P_{0}}^{2} \chi\right]\left[\omega^{2} / \gamma^{2}+2 p_{P_{0}}^{2} \chi\right]^{2}} \sigma_{\gamma}\left(T_{0} \rightarrow T_{F}\right) \\
& =\frac{2}{\pi} \int d \omega \frac{1}{\omega}\left[\ln \gamma-1 / 2+\theta\left(\frac{\omega^{2}}{p_{P_{0}}^{2}} \ln \frac{p_{P_{0}}}{\omega}\right)\right] \sigma_{\gamma}\left(T_{0} \rightarrow T_{F}\right)
\end{aligned}
$$


displaying the leading $\ln \gamma$ of the Weizsacker-Williams form. We do not attempt to recover the subsequent terms.

It is necessary to note the limitations inherent in the above derivation. In order to take over the photo cross section, the closeness of $|\bar{q}|$ to $\omega$ is vital. This is, in fact, correct for the part that leads to the $\ln \gamma$ dependence. It is, however, less true for the higher orders. In fact, for the $\chi^{2}$ term it is not at all valid, since if $\chi$ is replaced by $\chi^{2}$ the weighting is no longer such that $2 p_{P_{0}} p_{P_{f}} \chi \sim \mathcal{O}\left(\omega^{2} / \gamma^{2}\right)$, but is rather spread over $\mathcal{O}\left(\omega^{2}\right)$; then $\vec{q}^{2}$ is no longer just $\omega^{2}$, but $\omega^{2}+\mathcal{O}\left(\omega^{2}\right)$. This does not ruin the leading $(\ln \gamma)$ approximation since it can easily be seen that the involving term $\chi^{2}$ is small, of order $\omega^{2} / p_{P_{0}}^{2}\left(\ln \left(p_{P_{0}} / \omega\right) / \ln \gamma\right)$ relative to the leading term, if one makes the reasonable assumption that the matrix element, $\left\langle T_{F}\left|j_{\perp} e^{i \vec{q} \cdot \vec{r}}\right| T_{0}\right\rangle$, is of the same order for the larger range of $|\vec{q}|$. If, however, we wish to calculate some average value of momentum transfer, as say

$$
p_{P_{0}} \sin \theta \simeq \sqrt{2 \chi} p_{P_{0}}
$$

the extra weighting involved in the calculation of

$$
\int d \chi \frac{d \sigma}{d \Omega} \sqrt{\chi} / \int d \chi \frac{d \sigma}{d \Omega}
$$

moves $|\vec{q}|$ into the larger domain. Therefore, without bringing in the full form factor of the photo reaction no close approximation is possible. It can, however, provide an order of magnitude estimate. Four our purposes here, we need not go into it.

It is sufficient to note that the leading order of the excitation cross section is contained within the domain roughly specified by

$$
2 p_{P_{0}} p_{P_{f}} \chi \sim \mathcal{O}\left(\omega^{2} / \gamma^{2}\right)
$$

Since the transverse component is defined by

$$
p_{P_{\perp}}=p_{P} \sin \theta \sim p_{P} \theta
$$

we have that the effective range of transverse momenta is given as:

$$
p_{P_{\perp}}=p_{P} \sin \theta \sim \mathcal{O}(\omega / \gamma)
$$

the order of magnitude used in the text. 

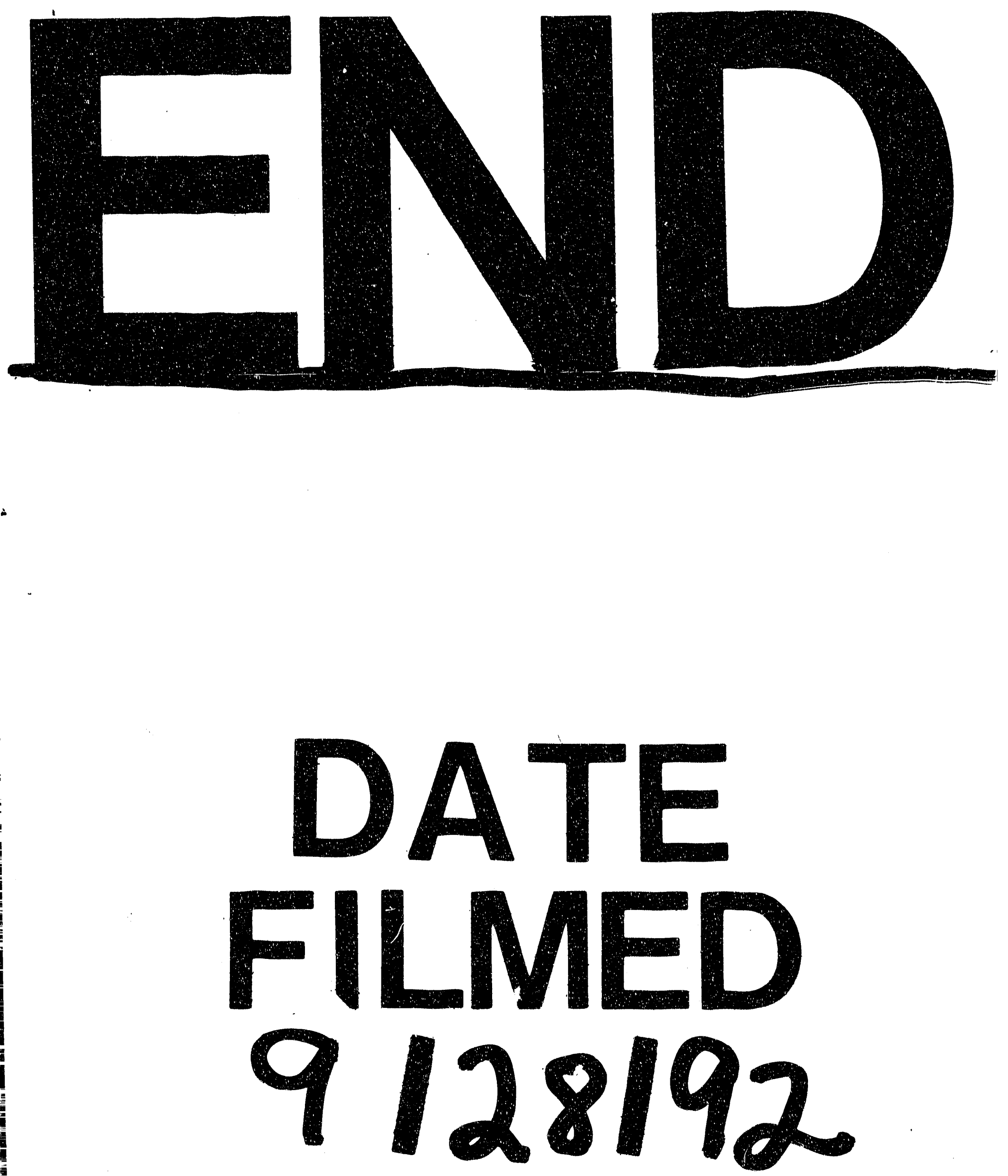
